\title{
Pharmacologic Weight Loss: An Underutilized Practice in the Fight Against Obesity
}

ISSN: 2578-0263

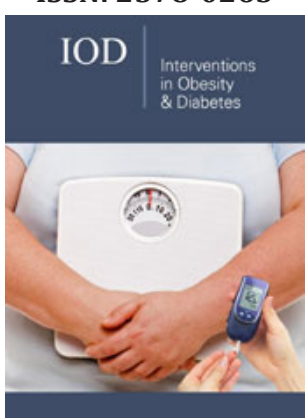

*Corresponding author: Megumi Howard, Nova Southeastern University College of Pharmacy, Davie, Florida

Submission: 泟 November 20, 2020

Published: 㳑January 19, 2021

Volume 4 - Issue 5

How to cite this article: Rizo M, Aguas Cabral M, Howard M. Pharmacologic Weight Loss: An Underutilized Practice in the Fight Against Obesity. Interventions Obes Diabetes 4(5). IOD.000596. 2021. DOI: 10.31031/IOD.2021.04.000596

Copyright@ Megumi Howard. This article is distributed under the terms of the Creative Commons Attribution 4.0 International License, which permits unrestricted use and redistribution provided that the original author and source are credited.

\author{
Rizo $\mathbf{M}^{1}$, Aguas Cabral M I ${ }^{2}$ and Howard $\mathbf{M}^{3 *}$ \\ ${ }^{1}$ PharmCare Services, Florida \\ ${ }^{2}$ Mercy Hospital, Florida \\ ${ }^{3}$ Nova Southeastern University College of Pharmacy, Florida
}

\begin{abstract}
Obesity is considered one of the most contemporary threats to non-communicable disease such as cardiovascular disease, diabetes, musculoskeletal disorders and even some types of cancers. Its worldwide prevalence has nearly tripled between 1975 and 2016. In 2016, more than 1.9 billion adults aged 18 years and older were categorized as overweight, and of these over 650 million adults were obese. However, Weight management medications (WMM) are currently underutilized as an adjunct to behavioral and lifestyle interventions. By way of example, only $2 \%$ of eligible veterans received prescriptions for pharmacologic weight loss in the 2014-2015 fiscal years, and up to 1\% of obese U.S. individuals filled a prescription for a WMM between 2009-2013. There are currently five FDA-approved medications for long-term weight loss medications. We analyzed 24 randomized clinical trials of the five drugs and interpreted findings. Of those 24, lorcaserin (Belviq ${ }^{\circledR}$ ), naltrexone and bupropion (Contrave ${ }^{\circledR}$ ), and phentermine and topiramate $(\mathrm{Qsymia} \circledast)$ ) had four studies each, while liraglutide (Saxenda $₫)$, and orlistat (Xenical ${ }^{\circledR}$ ) had six studies each. Underutilization of pharmacologic weight corrective therapies that have been statistically and clinically proven to be valuable tools in reducing obesity and its related risk factors. Studies of the five FDA-approved drugs have demonstrated clinically significant positive effects on weight loss with differing effects on both cardiovascular and glycemic markers/risk factors.
\end{abstract}

Keywords: Weight loss; Anti-obesity drugs; Obesity; Lorcaserin; Naltrexone and bupropion; Phentermine and topiramate;Liraglutide;Orlistat

Abbreviations: WHO: World Health Organization; MACE: Major Adverse Cardiovascular Events; BMI: Body Mass Index; HDL: High Density Lipoprotein; LDL: Low Density Lipoprotein

\section{Introduction}

\section{Obesity epidemic}

Obesity is considered one of the most serious contemporary threats to non-communicable diseases such as cardiovascular disease, diabetes, musculoskeletal disorders and even some types of cancers. Its worldwide prevalence has nearly tripled between 1975 and 2016. In 2016, more than 1.9 billion adults aged 18 years and older were categorized as overweight, and of these over 650 million adults were obese [1].

\section{Current practice recommendations}

The World Health Organization (WHO) has provided recommendations at both a societal and individual level: in order to reduce the obesity epidemic. At a societal level, WHO recommends supportive environments and communities that encourage healthy diet and exercise, whereas at the individual level, recommendations include limiting energy intake from total fats and sugars, increasing consumption of fruit and vegetables, as well as legumes, whole grains and nuts, and engaging in regular physical activity [1]. Thus, current first-line strategy for the treatment of obesity is through lifestyle interventions. Reducing in caloric intake to 500-1000 calories per day deficit, increasing physical activity to 150-200 minutes per week, and changes in health behaviors are core objectives. However, while lifestyle modification has long been considered a mainstay of therapy for obesity, it has only produced modest weight loss and no reduction in major adverse cardiovascular events (MACE) [2]. Many individuals are not able to achieve and maintain meaningful weight-loss though diet and exercise alone $[3,4]$. Possible explanations behind the insufficiency of diet and exercise alone are the human genetic component, compensatory behaviors, and poor perception of energy. To date, more than 400 different genes have been implicated in the causes of overweight or 
obesity, although only a handful appear to be major players. Genes contribute to obesity in many ways, by affecting appetite, satiety, metabolism, food cravings, body-fat distribution, and the tendency to use eating as a way to cope with stress. Compensatory behaviors refer to adjustments people may unconsciously make after working out to offset the calories burned. One 2009 study demonstrated that people seem to increase their food intake post-physical activity either because they thought they burned off a lot of calories or because they were hungrier. Another review of studies from 2012 found that people generally overestimated how much energy exercise burned and ate more when they worked out. Energy balance is expressed by the equation: ES (Energy Store): the rate of changed body energy stores equals EI (Energy Intake): the rate of energy intake minus EE (Energy Expenditure). The rate of energy expenditures typically expressed in kcal per day [5].

\section{Limitations to current practice quantitative measure- ments of overweight and obesity}

Body mass index (BMI), is used by WHO to measure body size and as an indicator of high body fatness. BMI is calculated using a person's weight in kilograms divided by the square of their height in meters. According to the WHO definition, overweight is a BMI greater than or equal to 25; and obesity is a BMI greater than or equal to 30. It is a quick and convenient population-level measure of overweight and obesity due to its universality: the calculation does not change between males and females, nor does it discriminate on age. However, despite its usefulness: BMI should be considered a rough guide rather than a true body fatness measure, as it is not powered to account for human variation in anatomy.

\section{The concept of clinically meaningful weight loss}

The idea of quantitative measurement of obesity came in stages. Quetelet introduced BMI, which was applied nearly a century later to the evaluation of degree of fatness in studies of familial inheritance of obesity [6]. Publication of average weight tables in the 1850s was expanded to "ideal" weight tables by the life insurance industry in the mid-20 $20^{\text {th }}$ century. However, the relation of increasing weight to disease risk was extended by the Framingham Study from which Gordon and Kannel concluded that if everyone were at optimal weight, the incidence of coronary heart disease would be reduced by $25 \%$ and congestive failure and brain infarctions would be reduced by 35\% [7]. By mid-1970's there had been many observations about the association of obesity and health issues. In 1973, the Fogarty Conference report suggested several criteria, including percent achieving 20- and 40-pound weight loss and a weight reduction index. Clearly the obvious question was, "What defines clinically significant weight loss?" Up to this point in time, few, if any, had suggested that modest weight losses might have important health benefits. In the 1980s, one approach to this question was based on defining clinically significant overweight as a body weight with a BMI $>30$. Thus, clinically significant weight loss would be reduction below a BMI of 30 .
By the early 1990s, Rossner interpreted outcomes of treatment by percent weight loss. He concluded that $<5 \%$ weight loss may reduce risk but was unsatisfactory, whereas a weight loss of 5-10\% was considered a "fair" response [8]. In 1992, Goldstein recommended $\leq 10 \%$ weight loss to define clinically meaningful weight loss [9]. Blackburn in 1995 suggested that 5\% might be a valid "single" criterion to assess significant weight loss [10]. Two landmark studies of diabetes prevention supported this recommendation. An average weight loss of $5.5 \%$ reduced the incidence of diabetes by $58 \%$ in the American Diabetes Prevention Program (ADPP) trial [11]. A systematic analysis of clinical trials with outcome data observed for at least 2 years by Douketis et al. [12] provided convincing evidence that $5 \%$ weight loss produced important improvements in risk factors or incidence of disease in populations "at risk" from their obesity [12]. A statistical model of the weight loss data from the ADPP trial by Hamman et al. [13] showed that for every kilogram of weight lost there was a $16 \%$ reduction in risk for progression to diabetes and that $5 \%$ weight loss would produce about $50 \%$ reduction in the incidence of type 2 diabetes [13]. Furthermore, a categorical analysis of weight loss from the Look AHEAD trial demonstrated a strong relationship between glycemic measures and weight loss, with improvement beginning at $2.5 \%$ to $5 \%$ weight loss. For systolic and diastolic blood pressure, high density lipoprotein (HDL) cholesterol, and triglycerides, improvement began at 5\% weight loss. In 2013, an expert panel formed by the National Institutes of Health (NIH) conducted an evidence based review of the literature around five critical questions [14]. Critical question one addressed the health benefits of weight loss: "What amount weight loss is necessary to achieve benefit with respect to cardiovascular disease (CVD) risk factors, morbidity, and mortality?" The graded evidence statements that resulted from this effort provide the strongest support for weight loss beginning at 3\% for glycemic measures and triglycerides, and 5\% for blood pressure and HDL and low density lipoprotein (LDL) cholesterol, to be considered clinically meaningful. The committee went on to conclude that increased amounts of weight loss provided even greater benefits. To achieve improvement in systolic and diastolic blood pressure, HDL and LDL cholesterol, $5 \%$ or more weight loss from baseline is considered meaningful, while for glycemic measures and triglycerides, $\geq 3 \%$ weight loss is considered clinically significant [15].

\section{Underutilization of pharmacologic corrective therapies}

Weight management medications (WMM) are currently underutilized as an adjunct to behavioral and lifestyle interventions. By way of example, only $2 \%$ of eligible veterans received prescriptions for pharmacologic weight loss in the 20142015 fiscal years, and up to $1 \%$ of obese U.S. individuals filled a prescription for a WMM between 2009-2013. In addition, physician prescribing patterns have been declining, with the exception of phentermine, since 1991. Possible explanations for the WMM prescribing downtrend include absence of physician training 
in pharmacotherapy for obesity and impediments by the U.S. healthcare system such as lack of insurance coverage or limited patient-provider time for discussing interventions [16,17].

\section{Examine the five US FDA-approved medications for long- term weight loss}

There are five FDA-approved weight loss medications for longterm use. See Table 1 for detail.

\section{Methods}

We analyzed 24 randomized clinical trials of the five weight loss drugs and interpreted findings. Of those 24, lorcaserin (Belviq ${ }^{\circledR}$ ), naltrexone and bupropion (Contrave ${ }^{\circledR}$ ), and phentermine and topiramate $\left(\mathrm{Q} s y m i{ }^{\circledR}\right)$ had four studies each, while liraglutide $\left(\right.$ Saxenda ${ }^{\circledR}$ ), and orlistat $\left(\right.$ Xenical $^{\circledR}$ ) had six studies each [18-22]. There were 21 double-blind, 22 placebo-controlled, 3 open-label and 11 multiple-arm studies evaluated. In addition, 54\% were post-market, while $38 \%$ and $8 \%$ were Phase III and Phase II trials, respectively. The published journals and numbers of trials are as follows: Obesity Journal: 5, International Journal of Obesity: 4, The Lancet: 3,JAMA: 3, Journal of Clinical Endocrinology and Metabolism: 2 , and 1 trial evaluated each for the Current Therapeutic Research Journal, Sleep Journal, Diabetes Care, New England Journal of Medicine, Diabetes, Obesity and Metabolism, Archives of Medical Science and American Journal of Clinical Nutrition. The summary of each characteristics of weight loss drugs are shown in Table 1.

Table 1: Five US FDA approved drugs for long-term weight loss.

\begin{tabular}{|c|c|c|c|c|c|}
\hline Adverse Effects & $\begin{array}{c}\text { Oily Spotting } \\
\text { Flatus with dis- } \\
\text { charge } \\
\text { Fecal urgency } \\
\text { Increased defecation } \\
\text { and fecal inconti- } \\
\text { nence }\end{array}$ & $\begin{array}{l}\text { Paraesthesia } \\
\text { Dizziness } \\
\text { Insomnia } \\
\text { Constipation } \\
\text { Dysgeusia } \\
\text { Constipation }\end{array}$ & $\begin{array}{l}\text { Headache } \\
\text { Dizziness } \\
\text { Nausea } \\
\text { Dry Mouth } \\
\text { Constipation } \\
\text { Hypoglycemia }\end{array}$ & $\begin{array}{l}\text { Headache } \\
\text { Dizziness } \\
\text { Nausea } \\
\text { Dry Mouth } \\
\text { Constipation } \\
\text { Insomnia }\end{array}$ & $\begin{array}{c}\text { Headache } \\
\text { Dizziness } \\
\text { Nausea } \\
\text { Dry Mouth } \\
\text { Constipation } \\
\text { Hypoglycemia }\end{array}$ \\
\hline Contraindications & $\begin{array}{c}\text { Pregnancy } \\
\text { Chronic Malabsorp- } \\
\text { tion Syndrome } \\
\text { Cholestasis } \\
\text { Known Hypersensi- } \\
\text { tivity to Xenical }{ }^{\circledR}\end{array}$ & $\begin{array}{c}\text { Pregnancy } \\
\text { Glaucoma } \\
\text { Hyperthyroidism } \\
\text { During or within } 14 \\
\text { days of monoamine } \\
\text { oxidase inhibitor } \\
\text { Known Hypersensi- } \\
\text { tivity or idiosyncrasy } \\
\text { to sympathomimetic } \\
\text { amines }\end{array}$ & Pregnancy & $\begin{array}{c}\text { Pregnancy } \\
\text { Uncontrolled HTN } \\
\text { Seizure disorders } \\
\text { Chronic Opioid } \\
\text { use } \\
\text { MAOI inhibitors }\end{array}$ & $\begin{array}{c}\text { Pregnancy } \\
\text { Hypersensitivity to } \\
\text { liraglutide or any } \\
\text { product components } \\
\text { Personal or family } \\
\text { history of medullary } \\
\text { thyroid carcinoma or } \\
\text { Multiple Endocrine } \\
\text { Neoplasia syndrome } \\
\text { type } 2\end{array}$ \\
\hline Indications & $\begin{array}{l}\text { For obesity man- } \\
\text { agement including } \\
\text { weight loss and } \\
\text { weight mainte- } \\
\text { nance when used in } \\
\text { conjunction with a } \\
\text { reduced-calorie diet } \\
\text { To reduce the risk } \\
\text { for weight regain } \\
\text { after prior weight } \\
\text { loss }\end{array}$ & $\begin{array}{l}\text { Adjunct to a re- } \\
\text { duced-caloric diet and } \\
\text { increased physical } \\
\text { activity for chronic } \\
\text { weight management } \\
\text { in adults with an } \\
\text { initial BMI of } \geq 30 \mathrm{~kg} / \\
\mathrm{m}^{2} \text { or } \geq 27 \mathrm{~kg} / \mathrm{m}^{2} \text { in the } \\
\text { presence of at least } \\
\text { one weight-related } \\
\text { co-morbidity such as } \\
\text { HTN, T2DM or DLD }\end{array}$ & $\begin{array}{c}\text { Adjunct to a } \\
\text { reduced-caloric } \\
\text { diet and in- } \\
\text { creased physical } \\
\text { activity for } \\
\text { chronic weight } \\
\text { management } \\
\text { in adults with } \\
\text { an initial BMI } \\
\text { of } \geq 30 \mathrm{~kg} / \mathrm{m}^{2} \\
\text { or } \geq 27 \mathrm{~kg} / \mathrm{m}^{2} \\
\text { in the presence } \\
\text { of at least one } \\
\text { weight-related } \\
\text { co-morbidity } \\
\text { such as HTN, } \\
\text { T2DM or DLD }\end{array}$ & $\begin{array}{l}\text { Adjunct to a re- } \\
\text { duced-caloric diet } \\
\text { and increased } \\
\text { physical activity } \\
\text { for chronic weight } \\
\text { management in } \\
\text { adults with an ini- } \\
\text { tial BMI of } \geq 30 \mathrm{~kg} / \\
\mathrm{m}^{2} \text { or } \geq 27 \mathrm{~kg} / \mathrm{m}^{2} \\
\text { in the presence } \\
\text { of at least one } \\
\text { weight-related } \\
\text { co-morbidity such } \\
\text { as HTN, T2DM or } \\
\text { DLD }\end{array}$ & $\begin{array}{l}\text { Adjunct to a re- } \\
\text { duced-caloric diet and } \\
\text { increased physical } \\
\text { activity for chronic } \\
\text { weight management } \\
\text { in adults with an } \\
\text { initial BMI of } \geq 30 \mathrm{~kg} / \\
\mathrm{m}^{2} \text { or } \geq 27 \mathrm{~kg} / \mathrm{m}^{2} \text { in the } \\
\text { presence of at least } \\
\text { one weight-related } \\
\text { co-morbidity such as } \\
\text { HTN, T2DM or DLD }\end{array}$ \\
\hline
\end{tabular}




\begin{tabular}{|c|c|c|c|c|c|}
\hline Dose & $\begin{array}{l}\text { One } 120 \text {-mg capsule } \\
\text { three times a day } \\
\text { with each fatty meal }\end{array}$ & $\begin{array}{l}\text { Take once daily in } \\
\text { morning. } \\
\text { Avoid evening dose to } \\
\text { avoid insomnia. }\end{array}$ & $\begin{array}{c}\text { Take 10mg twice } \\
\text { daily } \\
\text { D/C if } 5 \% \text { WL } \\
\text { not achieved by } \\
\text { week } 12\end{array}$ & $\begin{array}{c}\text { Week } 11 \text { tab qam, } \\
\text { week } 21 \text { tab BID, } \\
\text { week } 32 \text { tab qam } \\
1 \text { tab qpm, } \\
\text { week } 42 \text { tabs BID }\end{array}$ & $\begin{array}{l}\text { 3mg SQ daily. Initiate } \\
\text { at } 0.6 \mathrm{mg} \text { per day for } \\
\text { one week. In weekly } \\
\text { intervals increase the } \\
\text { dose until a dose of } \\
3 \mathrm{mg} \text { is reached. }\end{array}$ \\
\hline Drug Category & $\begin{array}{l}\text { Reversible Inhibitor } \\
\text { of Gastrointestinal } \\
\text { Lipases }\end{array}$ & $\begin{array}{l}\text { PHEN: Sympathomi- } \\
\text { metic amine anorectic } \\
\text { TOP: Antiepileptic }\end{array}$ & $\begin{array}{c}\text { Serotonin } 2 \mathrm{C} \\
\text { receptor agonist }\end{array}$ & $\begin{array}{l}\text { NAL: An opioid } \\
\text { antagonist } \\
\text { BUP: Aminoke- } \\
\text { tone antidepres- } \\
\text { sant }\end{array}$ & $\begin{array}{l}\text { Glucagon-like pep- } \\
\text { tide-1 (GLP-1) recep- } \\
\text { tor agonist }\end{array}$ \\
\hline $\begin{array}{c}\text { Drug } \\
\text { Approved Year }\end{array}$ & $\begin{array}{c}\text { Xenical }^{\circledR} \\
\text { (orlistat)18 } \\
1999\end{array}$ & $\begin{array}{c}\text { Qsymia }^{\circledR} \\
\text { (phentermine and } \\
\text { topiramate)19 } \\
2012\end{array}$ & $\begin{array}{c}\text { Belviq }^{\circledR} \\
\text { (lorcaserin)20 } \\
2012\end{array}$ & $\begin{array}{l}\text { Contrave }^{\circledR} \\
\text { (naltrexone and } \\
\text { bupropion)21 } \\
2014\end{array}$ & $\begin{array}{c}\text { Saxenda }^{\circledR} \\
\text { (liraglutide)22 } \\
2014\end{array}$ \\
\hline
\end{tabular}

\section{Results}

\section{Xenical ${ }^{\circledR}$ (orlistat)}

We selected six Xenical ${ }^{\circledR}$ studies for review, conducted by Davidson et al. [23-28] respectively (Table 2). 23-28 Five out of 6 studies demonstrated statistically significant weight loss and four out of six Xenical studies demonstrated clinically significant weight loss. For waist circumference measurement, two out of three Xenical studies led to statistically significant values versus control. Two out of two studies demonstrated statistically significant reduction in blood pressure while one out of two studies demonstrated statistically significant differences in fasting blood sugar versus control. In regard to cholesterol, four out of four studies demonstrated statistically significant reductions in total and LDL cholesterol, however, non-statistically significant variation was noted with triglycerides. Unfavorable yet statistically significant increase in HDL cholesterol was seen in comparison of Xenical and control group (Table 3).

Table 2: Selected studies for Xenical®.

\begin{tabular}{|c|c|c|c|c|}
\hline Primary Author & Year & Journal & Sample Size & Study Length \\
\hline Davidson M.H. & 1999 & JAMA & 892 Participants & 24 Months \\
\hline Finer N. & 2000 & International Journal of Obesity & 218 Participants & 12 Months \\
\hline Muls E. & 2001 & International Journal of Obesity & 290 Participants & 6 Months \\
\hline Ozcelik 0. & 2004 & Current Therapeutic Research & 24 Participants & 3 Months \\
\hline Torgerson J.S. & 2004 & Diabetes Care & 3305 Participants & 48 Months \\
\hline Kuhawska-tuczak M. & 2017 & Archives of Medical Science & 73 Participants & 3 Months \\
\hline
\end{tabular}

Table 3: Results of studies for Xenical ${ }^{\circledR}$.

\begin{tabular}{|c|c|c|c|}
\hline Primary Author & D WT Difference & C WT Difference & WT B/W Group P-value \\
\hline Kuhawska-tuczak [28] & $-9.4 \mathrm{~kg}$ & $-4.9 \mathrm{~kg}$ & $\leq 0.05$ \\
\hline Ozcelik [26] & $-6.76 \mathrm{~kg}$ & $-9 \mathrm{~kg}$ & NS \\
\hline Torgerson [27] & $-5.8 \mathrm{~kg}$ & $-3 \mathrm{~kg}$ & $\leq 0.001$ \\
\hline Finer [24] & $-3.29 \mathrm{~kg}$ & $-1.31 \mathrm{~kg}$ & $\leq 0.01$ \\
\hline Muls [25] & $-4.66 \mathrm{~kg}$ & $-1.88 \mathrm{~kg}$ & $\leq 0.001$ \\
\hline Davidson [23] & $-3.2 \mathrm{~kg}$ & $5.63 \mathrm{~kg}$ & $\leq 0.001$ \\
\hline Primary Author & D WC Difference & C WC Difference & WC B/W Group P value \\
\hline Kuhawska-tuczak [28] & $-10.5 \mathrm{~cm}$ & $-5.8 \mathrm{~cm}$ & $\leq 0.05$ \\
\hline Torgerson [27] & $-6.4 \mathrm{~cm}$ & $-4.4 \mathrm{~cm}$ & $\leq 0.01$ \\
\hline Finer [24] & $-6.3 \mathrm{~cm}$ & $-5.1 \mathrm{~cm}$ & NS \\
\hline
\end{tabular}




\begin{tabular}{|c|c|c|c|}
\hline Primary Author & D SBP Difference & C SBP Difference & SBP B/W Group P value \\
\hline Torgerson [27] & $-4.9 \mathrm{mmHg}$ & $-3.4 \mathrm{mmHg}$ & $\leq 0.01$ \\
\hline Davidson [23] & $-0.8 \mathrm{mmHg}$ & $1 \mathrm{mmHg}$ & $\leq 0.01$ \\
\hline Primary Author & D DBP Difference & C DBP Difference & DBP B/W Group P value \\
\hline Torgerson [27] & $-2.6 \mathrm{mmHg}$ & $-1.9 \mathrm{mmHg}$ & $\leq 0.01$ \\
\hline Davidson [23] & $-0.5 \mathrm{mmHg}$ & $1.3 \mathrm{mmHg}$ & $\leq 0.01$ \\
\hline Primary Author & D FBG Difference & C FBG Difference & FBG B/W Group P value \\
\hline Kuhawska-tuczak [28] & $0.31 \mathrm{mg} / \mathrm{dL}$ & $0.09 \mathrm{mg} / \mathrm{dL}$ & NS \\
\hline Torgerson [27] & $1.8 \mathrm{mg} / \mathrm{dL}$ & $3.6 \mathrm{mg} / \mathrm{dL}$ & $\leq 0.01$ \\
\hline Primary Author & D TC Difference & C TC Difference & TC B/W Group P value \\
\hline Torgerson [27] & $-17.72 \mathrm{mg} / \mathrm{dL}$ & $-5.16 \mathrm{mg} / \mathrm{dL}$ & $\leq 0.01$ \\
\hline Finer [24] & $-1.93 \mathrm{mg} / \mathrm{dL}$ & $11.6 \mathrm{mg} / \mathrm{dL}$ & $\leq 0.001$ \\
\hline Muls [25] & $-16.24 \mathrm{mg} / \mathrm{dL}$ & $5.41 \mathrm{mg} / \mathrm{dL}$ & $\leq 0.001$ \\
\hline Davidson [23] & $-5.02 \mathrm{mg} / \mathrm{dL}$ & $3.09 \mathrm{mg} / \mathrm{dL}$ & $\leq 0.05$ \\
\hline Primary Author & D LDL Difference & C LDL Difference & LDL B/W Group P value \\
\hline Torgerson [27] & $-18.31 \mathrm{mg} / \mathrm{dL}$ & $-7.49 \mathrm{mg} / \mathrm{dL}$ & $\leq 0.01$ \\
\hline Finer [24] & $-4.25 \mathrm{mg} / \mathrm{dL}$ & $8.12 \mathrm{mg} / \mathrm{dL}$ & $\leq 0.001$ \\
\hline Muls [25] & $-20.5 \mathrm{mg} / \mathrm{dL}$ & $-3.48 \mathrm{mg} / \mathrm{dL}$ & $\leq 0.001$ \\
\hline Davidson [23] & $-5.41 \mathrm{mg} / \mathrm{dL}$ & $8.9 \mathrm{mg} / \mathrm{dL}$ & $\leq 0.05$ \\
\hline Primary Author & D HDL Difference & C HDL Difference & HDL B/W Group P value \\
\hline Torgerson [27] & $3 \mathrm{mg} / \mathrm{dL}$ & $4.2 \mathrm{mg} / \mathrm{dL}$ & 0.01 \\
\hline Finer [24] & $1.11 \mathrm{mg} / \mathrm{dL}$ & $1.08 \mathrm{mg} / \mathrm{dL}$ & NS \\
\hline Muls [25] & $2.7 \mathrm{mg} / \mathrm{dL}$ & $6.2 \mathrm{mg} / \mathrm{dL}$ & 0.001 \\
\hline Primary Author & D TG Difference & C TG Difference & TG B/W Group P value \\
\hline Torgerson [27] & $4.04 \mathrm{mg} / \mathrm{dL}$ & $4.89 \mathrm{mg} / \mathrm{dL}$ & NS \\
\hline Muls [25] & $7.09 \mathrm{mg} / \mathrm{dL}$ & $12.4 \mathrm{mg} / \mathrm{dL}$ & NS \\
\hline
\end{tabular}

\section{Qsymia ${ }^{\circledR}$ (phentermine and topiramate)}

For Qsymia ${ }^{\circledR}$, we reviewed four studies conducted by Allison et al. [29-32] \& (Table 4). Four out of four Qsymia studies demonstrated statistically and clinically significant weight loss and three out of three studies measuring waist circumference led to statistically significant values compared to control group. Three out of four studies demonstrated statistically significant reductions in systolic blood pressure, two out of four demonstrated statistically significant reductions in fasting blood sugar and one demonstrated significant reductions in C-reactive protein. For cholesterol reduction, two out of three studies demonstrated statistically significant differences in total cholesterol, one out of four studies demonstrated unfavorable statistically significant differences in LDL cholesterol. Two out of four demonstrated statistically significant differences in HDL cholesterol (one favorable and one unfavorable), and two out of four studies demonstrated statistically significant differences in triglycerides. All results are summarized in Table 5.

Table 4: Selected studies for Qsymia ${ }^{\circledR}$.

\begin{tabular}{|c|c|c|c|c|}
\hline Primary Author & Year & Journal & Sample Size & Study Length \\
\hline Allison D.B. & 2011 & Obesity Journal & 1269 Participants & 14 Months \\
\hline Gadde K.M. & 2011 & The Lancet & 2487 Participants & 14 Months \\
\hline Garvey W.T. & 2012 & American Journal of Clinical Nutrition & 676 Participants & 14 Months \\
\hline Winslow D.H. & 2012 & Sleep Journal & 45 Participants & 7 Months \\
\hline
\end{tabular}


Table 5: Results of studies for Qsymia ${ }^{\circledR}$.

\begin{tabular}{|c|c|c|c|}
\hline Primary Author & Drug WT Difference & Control WT Difference & WT B/W Group P-value \\
\hline Garvey [31] & $-9.495 \mathrm{~kg}$ & $-1.82 \mathrm{~kg}$ & $\leq 0.0001$ \\
\hline Allison [29] & $-6.04 \mathrm{~kg}$ & $-1.795 \mathrm{~kg}$ & $\leq 0.0001$ \\
\hline Winslow [32] & $-11 \mathrm{~kg}$ & $-4.5 \mathrm{~kg}$ & $\leq 0.001$ \\
\hline Gadde [30] & $-8.1 \mathrm{~kg}$ & $-1.4 \mathrm{~kg}$ & $\leq 0.0001$ \\
\hline Primary Author & Drug WC Difference & Control WC Difference & WC B/W Group P value \\
\hline Garvey [31] & $-9.8 \mathrm{~cm}$ & $-3.6 \mathrm{~cm}$ & $\leq 0.0001$ \\
\hline Allison [29] & $-5.6 \mathrm{~cm}$ & $-3.1 \mathrm{~cm}$ & $\leq 0.001$ \\
\hline Gadde [30] & $-7.6 \mathrm{~cm}$ & $-2.4 \mathrm{~cm}$ & $\leq 0.0001$ \\
\hline Primary Author & Drug WC Difference & Control WC Difference & WC B/W Group P value \\
\hline Garvey [31] & $-9.8 \mathrm{~cm}$ & $-3.6 \mathrm{~cm}$ & $\leq 0.0001$ \\
\hline Allison [29] & $-5.6 \mathrm{~cm}$ & $-3.1 \mathrm{~cm}$ & $\leq 0.001$ \\
\hline Gadde [30] & $-7.6 \mathrm{~cm}$ & $-2.4 \mathrm{~cm}$ & $\leq 0.0001$ \\
\hline Primary Author & Drug SBP Difference & Control SBP Difference & SBP B/W Group P value \\
\hline Garvey [31] & $-4.7 \mathrm{mmHg}$ & $-3.2 \mathrm{mmHg}$ & NS \\
\hline Allison [29] & $-1.8 \mathrm{mmHg}$ & $-2.9 \mathrm{mmHg}$ & $\leq 0.001$ \\
\hline Winslow [32] & $-15 \mathrm{mmHg}$ & $-7.3 \mathrm{mmHg}$ & $\leq 0.05$ \\
\hline Gadde [30] & $-4.7 \mathrm{mmHg}$ & $-2.4 \mathrm{mmHg}$ & $\leq 0.001$ \\
\hline Primary Author & Drug DBP Difference & Control DBP Difference & DBP B/W Group P value \\
\hline Garvey [31] & $-3.7 \mathrm{mmHg}$ & $-3.9 \mathrm{mmHg}$ & NS \\
\hline Allison [29] & $-0.1 \mathrm{mmHg}$ & $0.4 \mathrm{mmHg}$ & NS \\
\hline Winslow [32] & $-6.3 \mathrm{mmHg}$ & $-5.6 \mathrm{mmHg}$ & NS \\
\hline Gadde [30] & $-3.4 \mathrm{mmHg}$ & $-2.7 \mathrm{mmHg}$ & NS \\
\hline Primary Author & Drug FBG Difference & Control FBG Difference & FBG B/W Group P value \\
\hline Garvey [31] & $0.1 \mathrm{mg} / \mathrm{dL}$ & $3.7 \mathrm{mg} / \mathrm{dL}$ & NS \\
\hline Allison [29] & $-1.6 \mathrm{mg} / \mathrm{dL}$ & $1.9 \mathrm{mg} / \mathrm{dL}$ & $\leq 0.0001$ \\
\hline Winslow [32] & $-8.9 \mathrm{mg} / \mathrm{dL}$ & $-5.6 \mathrm{mg} / \mathrm{dL}$ & NS \\
\hline Gadde [30] & $-0.18 \mathrm{mg} / \mathrm{dL}$ & $2.34 \mathrm{mg} / \mathrm{dL}$ & $\leq 0.005$ \\
\hline Primary Author & Drug hs-CRP Difference & Control hs-CRP Difference & hs-CRP B/W Group P value \\
\hline Gadde [30] & $-2.49 \mathrm{mg} / \mathrm{dL}$ & $-0.79 \mathrm{mg} / \mathrm{dL}$ & $\leq 0.0001$ \\
\hline Primary Author & Drug TC Difference & Control TC Difference & TC B/W Group P value \\
\hline Allison [29] & $-10.589 \mathrm{mg} / \mathrm{dL}$ & $-6.79 \mathrm{mg} / \mathrm{dL}$ & $\leq 0.05$ \\
\hline Winslow [32] & $-13.9 \mathrm{mg} / \mathrm{dL}$ & $-6.2 \mathrm{mg} / \mathrm{dL}$ & NS \\
\hline Gadde [30] & $-9.85 \mathrm{mg} / \mathrm{dL}$ & $-6.76 \mathrm{mg} / \mathrm{dL}$ & $\leq 0.05$ \\
\hline Primary Author & Drug LDL Difference & Control LDL Difference & LDL B/W Group P value \\
\hline Garvey [31] & $-5.598 \mathrm{mg} / \mathrm{dL}$ & $-13.172 \mathrm{mg} / \mathrm{dL}$ & $\leq 0.05$ \\
\hline Allison [29] & $-9.43 \mathrm{mg} / \mathrm{dL}$ & $-6.67 \mathrm{mg} / \mathrm{dL}$ & NS \\
\hline Winslow [32] & $-11 \mathrm{mg} / \mathrm{dL}$ & $-1.6 \mathrm{mg} / \mathrm{dL}$ & NS \\
\hline Gadde [30] & $-4.44 \mathrm{mg} / \mathrm{dL}$ & $-5.07 \mathrm{mg} / \mathrm{dL}$ & NS \\
\hline Primary Author & Drug HDL Difference & Control HDL Difference & HDL B/W Group P value \\
\hline Garvey [31] & $3.55 \mathrm{mg} / \mathrm{dL}$ & $2.33 \mathrm{mg} / \mathrm{dL}$ & NS \\
\hline Allison [29] & $0.251 \mathrm{mg} / \mathrm{dL}$ & $0 \mathrm{mg} / \mathrm{dL}$ & NS \\
\hline Winslow [32] & $-1.1 \mathrm{mg} / \mathrm{dL}$ & $2.3 \mathrm{mg} / \mathrm{dL}$ & $\leq 0.05$ \\
\hline Gadde [30] & $2.61 \mathrm{mg} / \mathrm{dL}$ & $0.6 \mathrm{mg} / \mathrm{dL}$ & $\leq 0.0001$ \\
\hline
\end{tabular}




\begin{tabular}{|c|c|c|c|}
\hline Primary Author & Drug TG Difference & Control TG Difference & TG B/W Group P value \\
\hline Garvey [31] & $-19.65 \mathrm{mg} / \mathrm{dL}$ & $0.6176 \mathrm{mg} / \mathrm{dL}$ & $\leq 0.001$ \\
\hline Allison [29] & $6.068 \mathrm{mg} / \mathrm{dL}$ & $10.81 \mathrm{mg} / \mathrm{dL}$ & NS \\
\hline Winslow [32] & $-32.4 \mathrm{mg} / \mathrm{dL}$ & $-33 \mathrm{mg} / \mathrm{dL}$ & NS \\
\hline Gadde [30] & $-13.71 \mathrm{mg} / \mathrm{dL}$ & $7.49 \mathrm{mg} / \mathrm{dL}$ & $\leq 0.0001$ \\
\hline
\end{tabular}

\section{Belviq (lorcaserin)}

We reviewed four Belviq ${ }^{\circledR}$ clinical studies conducted by Fidler et al. [33-36] \& (Table 6). Three out of four studies demonstrated statistically and clinically significant weight loss, and two out of three studies measuring waist circumference led to statistically significant values versus control. One out of four studies demonstrated statistically significant reductions in blood pressure and significant fasting blood sugar. No difference has been seen on C-reactive protein value while one out of three studies demonstrated statistically significant differences in triglycerides. Results are shown in Table 7.

Table 6: Selected studies for Belviq ${ }^{\circledR}$.

\begin{tabular}{|c|c|c|c|c|}
\hline Primary Author & Year & Journal & Sample Size & Study Length \\
\hline Fidler M.C. & 2011 & Journal of Clinical Endocrinology and Metabolism & 4004 Participants & 13 Months \\
\hline O'Neil P.M. & 2012 & Obesity Journal & 603 Participants & 24 Months \\
\hline Smith S.R. & 2017 & Obesity Journal & 238 Participants & 3 Months \\
\hline Tronieri J.S. & 2018 & Obesity Journal & 137 Participants & 12 Months \\
\hline
\end{tabular}

Table 7: Results of studies for Belviq ${ }^{\circledR}$.

\begin{tabular}{|c|c|c|c|}
\hline Primary Author & Drug WT Difference & Control WT Difference & WT B/W Group P-value \\
\hline Fidler [33] & $-5.8 \mathrm{~kg}$ & $-2.9 \mathrm{~kg}$ & $\leq 0.001$ \\
\hline Tronieri [36] & $-8.4 \mathrm{~kg}$ & $-6.1 \mathrm{~kg}$ & NS \\
\hline Smith [35] & $-3.5 \mathrm{~kg}$ & $-7 \mathrm{~kg}$ & $\leq 0.05$ \\
\hline O’Neil [34] & $-4.7 \mathrm{~kg}$ & $-1.6 \mathrm{~kg}$ & $\leq 0.001$ \\
\hline Primary Author & Drug WC Difference & Control WC Difference & WC B/W Group P value \\
\hline Fidler [33] & $-6.3 \mathrm{~cm}$ & $-4.1 \mathrm{~cm}$ & $\leq 0.001$ \\
\hline Smith [35] & $-3.4 \mathrm{~cm}$ & $-4.7 \mathrm{~cm}$ & NM \\
\hline O'Neil [34] & $-5.5 \mathrm{~cm}$ & $-3.3 \mathrm{~cm}$ & $\leq 0.001$ \\
\hline Primary Author & Drug SBP Difference & Control SBP Difference & SBP B/W Group P value \\
\hline Fidler [33] & $-1.9 \mathrm{mmHg}$ & $-1.2 \mathrm{mmHg}$ & NS \\
\hline Tronieri [36] & $-1.6 \mathrm{mmHg}$ & $-7.6 \mathrm{mmHg}$ & NS \\
\hline Smith [35] & $-5.5 \mathrm{mmHg}$ & $-3.3 \mathrm{mmHg}$ & $\leq 0.05$ \\
\hline O’Neil [34] & $-0.8 \mathrm{mmHg}$ & $-0.9 \mathrm{mmHg}$ & NS \\
\hline Primary Author & Drug DBP Difference & Control DBP Difference & DBP B/W Group P value \\
\hline Fidler [33] & $-1.9 \mathrm{mmHg}$ & $-1.4 \mathrm{mmHg}$ & NS \\
\hline Tronieri [36] & $-1.4 \mathrm{mmHg}$ & $-2.6 \mathrm{mmHg}$ & NS \\
\hline Smith [35] & $-2.5 \mathrm{mmHg}$ & $-1.4 \mathrm{mmHg}$ & $\leq 0.05$ \\
\hline O’Neil [34] & $-1.1 \mathrm{mmHg}$ & $-0.7 \mathrm{mmHg}$ & NS \\
\hline Primary Author & Drug FBG Difference & Control FBG Difference & FBG B/W Group P value \\
\hline Tronieri [36] & $1.4 \mathrm{mg} / \mathrm{dL}$ & $4 \mathrm{mg} / \mathrm{dL}$ & NS \\
\hline O’Neil [34] & $-27.4 \mathrm{mg} / \mathrm{dL}$ & $-11.9 \mathrm{mg} / \mathrm{dL}$ & $\leq 0.001$ \\
\hline Primary Author & Drug hs-CRP Difference & Control hs-CRP Difference & hs-CRP B/W Group P value \\
\hline Tronieri [36] & $-2.6 \mathrm{mg} / \mathrm{dL}$ & $-1.7 \mathrm{mg} / \mathrm{dL}$ & NS \\
\hline O'Neil [34] & $-1.3 \mathrm{mg} / \mathrm{dL}$ & $-0.6 \mathrm{mg} / \mathrm{dL}$ & NS \\
\hline
\end{tabular}




\begin{tabular}{|c|c|c|c|}
\hline Primary Author & Drug TC Difference & Control TC Difference & TC B/W Group P value \\
\hline Fidler [33] & $-0.7 \mathrm{mg} / \mathrm{dL}$ & $0 \mathrm{mg} / \mathrm{dL}$ & NS \\
\hline Tronieri [36] & $-7.8 \mathrm{mg} / \mathrm{dL}$ & $-7.5 \mathrm{mg} / \mathrm{dL}$ & NS \\
\hline O'Neil [34] & $-0.7 \mathrm{mg} / \mathrm{dL}$ & $-1.7 \mathrm{mg} / \mathrm{dL}$ & NS \\
\hline Primary Author & Drug LDL Difference & Control LDL Difference & LDL B/W Group P value \\
\hline Fidler [33] & $0.3 \mathrm{mg} / \mathrm{dL}$ & $1.7 \mathrm{mg} / \mathrm{dL}$ & NS \\
\hline Tronieri [36] & $-8 \mathrm{mg} / \mathrm{dL}$ & $-6.1 \mathrm{mg} / \mathrm{dL}$ & NS \\
\hline O'Neil [34] & $4.2 \mathrm{mg} / \mathrm{dL}$ & $5 \mathrm{mg} / \mathrm{dL}$ & TG B/W Group P value \\
\hline Primary Author & Drug TG Difference & Control TG Difference & $\leq 0.05$ \\
\hline Fidler [33] & $-4.3 \mathrm{mg} / \mathrm{dL}$ & $-0.9 \mathrm{mg} / \mathrm{dL}$ & NS \\
\hline Tronieri [36] & $-5.5 \mathrm{mg} / \mathrm{dL}$ & $-5.3 \mathrm{mg} / \mathrm{dL}$ & NS \\
\hline O'Neil [34] & $-10.7 \mathrm{mg} / \mathrm{dL}$ & $-4.8 \mathrm{mg} / \mathrm{dL}$ & \\
\hline
\end{tabular}

\section{Contrave ${ }^{\circledR}$ (naltrexone and bupropion)}

We selected four Contrave ${ }^{\circledR}$ studies for review conducted by Greenway et al. [37-40] \& (Table 8). Four out of four studies demonstrated statistically and clinically significant weight loss, and four out of four studies measuring waist circumference led to statistically significant values versus control. Two out of three studies demonstrated statistically significant reductions in blood pressure, two out of three demonstrated statistically significant reductions in fasting blood sugar and two studies demonstrated significant reductions in C-reactive protein. One study demonstrated total cholesterol reduction and one out of three studies demonstrated statistically significant reduction in LDL cholesterol. Two out of three studies demonstrated statistically significant differences in HDL cholesterol while three out of three studies demonstrated statistically significant differences in triglycerides. Results are summarized in Table 9.

Table 8: Selected studies for Contrave ${ }^{\circledR}$.

\begin{tabular}{|c|c|c|c|c|}
\hline Primary Author & Year & Journal & Sample Size & Study Length \\
\hline Greenway F.L. & 2009 & Journal of Clinical Endocrinology and Metabolism & 203 Participants & 12 Months \\
\hline Greenway F.L. & 2010 & The Lancet & 1742 Participants & 14 Months \\
\hline Apovian C.M. & 2013 & Obesity Journal & 1496 Participants & 14 Months \\
\hline Nissen S.E. & 2016 & JAMA & 8905 Participants & 48 Months \\
\hline
\end{tabular}

Table 9: Results of studies for Contrave ${ }^{\circledR}$.

\begin{tabular}{|c|c|c|c|}
\hline Primary Author & Drug WT Difference & Control WT Difference & WT B/W Group P-value \\
\hline Nissen [40] & $-3.9 \mathrm{~kg}$ & $-1.2 \mathrm{~kg}$ & $\leq 0.001$ \\
\hline Greenway [38] & $-5.1 \mathrm{~kg}$ & $-0.9 \mathrm{~kg}$ & $\leq 0.05$ \\
\hline Apovian [39] & $-6.2 \mathrm{~kg}$ & $-1.3 \mathrm{~kg}$ & $\leq 0.001$ \\
\hline Greenway [37] & $-6.1 \mathrm{~kg}$ & $-1.3 \mathrm{~kg}$ & $\leq 0.0001$ \\
\hline Primary Author & Drug WC Difference & Control WC Difference & WC B/W Group P value \\
\hline Nissen [40] & $-2.1 \mathrm{~cm}$ & $-0.8 \mathrm{~cm}$ & $\leq 0.001$ \\
\hline Greenway [38] & $-5.4 \mathrm{~cm}$ & $-1 \mathrm{~cm}$ & $\leq 0.05$ \\
\hline Apovian [39] & $-6.7 \mathrm{~cm}$ & $-2.1 \mathrm{~cm}$ & $\leq 0.001$ \\
\hline Greenway [37] & $-6.2 \mathrm{~cm}$ & $-2.5 \mathrm{~cm}$ & $\leq 0.0001$ \\
\hline Primary Author & Drug SBP Difference & Control SBP Difference & SBP B/W Group P value \\
\hline Greenway [38] & $-1.6 \mathrm{mmHg}$ & $-1 \mathrm{mmHg}$ & NS \\
\hline Apovian [39] & $0.6 \mathrm{mmHg}$ & $-0.5 \mathrm{mmHg}$ & $\leq 0.05$ \\
\hline Greenway [37] & $-0.1 \mathrm{mmHg}$ & $-1.9 \mathrm{mmHg}$ & $\leq 0.001$ \\
\hline Primary Author & Drug DBP Difference & Control DBP Difference & DBP B/W Group P value \\
\hline Greenway [38] & $-1.2 \mathrm{mmHg}$ & $-4.4 \mathrm{mmHg}$ & NS \\
\hline Apovian [39] & $0.4 \mathrm{mmHg}$ & $0.3 \mathrm{mmHg}$ & NS \\
\hline
\end{tabular}




\begin{tabular}{|c|c|c|c|}
\hline Greenway [37] & $0 \mathrm{mmHg}$ & $-0.9 \mathrm{mmHg}$ & $\leq 0.01$ \\
\hline Primary Author & Drug FBG Difference & Control FBG Difference & FBG B/W Group P value \\
\hline Greenway [38] & $-2 \mathrm{mg} / \mathrm{dL}$ & $1.9 \mathrm{mg} / \mathrm{dL}$ & NS \\
\hline Apovian [39] & $-2.8 \mathrm{mg} / \mathrm{dL}$ & $-1.3 \mathrm{mg} / \mathrm{dL}$ & $\leq 0.05$ \\
\hline Greenway [37] & $-3.24 \mathrm{mg} / \mathrm{dL}$ & $-1.26 \mathrm{mg} / \mathrm{dL}$ & $\leq 0.0001$ \\
\hline Primary Author & Drug hs-CRP Difference & Control hs-CRP Difference & hs-CRP B/W Group P value \\
\hline Apovian [39] & $-1.09 \mathrm{mg} / \mathrm{dL}$ & $-0.307 \mathrm{mg} / \mathrm{dL}$ & $\leq 0.001$ \\
\hline Greenway [37] & $-1.11 \mathrm{mg} / \mathrm{dL}$ & $-0.596 \mathrm{mg} / \mathrm{dL}$ & $\leq 0.01$ \\
\hline Primary Author & Drug TC Difference & Control TC Difference & TC B/W Group P value \\
\hline Greenway [38] & $-9.5 \mathrm{mg} / \mathrm{dL}$ & $0.1 \mathrm{mg} / \mathrm{dL}$ & $\leq 0.05$ \\
\hline Primary Author & Drug LDL Difference & Control LDL Difference & LDL B/W Group P value \\
\hline Greenway [38] & $-4.3 \mathrm{mg} / \mathrm{dL}$ & $0.3 \mathrm{mg} / \mathrm{dL}$ & NS \\
\hline Apovian [39] & $-6.2 \mathrm{mg} / \mathrm{dL}$ & $-2.1 \mathrm{mg} / \mathrm{dL}$ & $\leq 0.01$ \\
\hline Greenway [37] & $-4.25 \mathrm{mg} / \mathrm{dL}$ & $-3.09 \mathrm{mg} / \mathrm{dL}$ & NS \\
\hline Primary Author & Drug HDL Difference & Control HDL Difference & HDL B/W Group P value \\
\hline Greenway [38] & $3.5 \mathrm{mg} / \mathrm{dL}$ & $1 \mathrm{mg} / \mathrm{dL}$ & NS \\
\hline Apovian [39] & $-0.9 \mathrm{mg} / \mathrm{dL}$ & $3.6 \mathrm{mg} / \mathrm{dL}$ & $\leq 0.001$ \\
\hline Greenway [37] & $0.09 \mathrm{mg} / \mathrm{dL}$ & $0 \mathrm{mg} / \mathrm{dL}$ & $\leq 0.0001$ \\
\hline Primary Author & Drug TG Difference & Control TG Difference & TG B/W Group P value \\
\hline Greenway [38] & $-43.6 \mathrm{mg} / \mathrm{dL}$ & $-15 \mathrm{mg} / \mathrm{dL}$ & $\leq 0.05$ \\
\hline Apovian [39] & $-11.65 \mathrm{mg} / \mathrm{dL}$ & $-0.564 \mathrm{mg} / \mathrm{dL}$ & $\leq 0.001$ \\
\hline Greenway [37] & $-14.79 \mathrm{mg} / \mathrm{dL}$ & $-3.45 \mathrm{mg} / \mathrm{dL}$ & $\leq 0.0001$ \\
\hline
\end{tabular}

\section{Saxenda ${ }^{\circledR}$ (liraglutide)}

We reviewed six studies for Saxenda ${ }^{\circledR}$ conducted by Wadden et al. [41-46] \& (Table 10). Five out of six studies demonstrated statistically and clinically significant weight loss, and five out of six studies measuring waist circumference led to statistically significant values versus control six. Five out of five studies demonstrated statistically significant reductions in systolic blood pressure, five out of six studies demonstrated statistically significant reductions in fasting blood sugar, and all six studies demonstrated significant reductions in C-reactive protein. Two out of three Saxenda ${ }^{\circledR}$ studies demonstrated statistically significant differences in total and HDL cholesterol while one out of three studies demonstrated statistically significant differences in LDL cholesterol. Three out of three studies demonstrated statistically significant differences in triglycerides. Results are shown in Table 11.

Table 10: Selected studies for Saxenda ${ }^{\circledR}$.

\begin{tabular}{|c|c|c|c|c|}
\hline Primary Author & Year & Journal & Sample Size & Study Length \\
\hline Wadden T.A. & 2013 & International Journal of Obesity & 422 Participants & 14 Months \\
\hline Davies M.J. & 2015 & JAMA & 846 Participants & 14 Months \\
\hline Pi-Sunyer X. & 2015 & New England Journal of Medicine & 3662 Participants & 14 Months \\
\hline Blackman A. & 2016 & International Journal of Obesity & 355 Participants & 8 Months \\
\hline Khoo J. & 2017 & Diabetes, Obesity and Metabolism & 24 Participants & 6.5 Months \\
\hline Le Roux C.W. & 2017 & The Lancet & 2210 Participants & 14 Months \\
\hline
\end{tabular}

Table 11: Results of studies for Saxenda $a^{\circledR}$.

\begin{tabular}{|c|c|c|c|}
\hline Primary Author & Drug WT Difference & Control WT Difference & WT B/W Group P-value \\
\hline Khoo [45] & $-3.5 \mathrm{~kg}$ & $-3.5 \mathrm{~kg}$ & NS \\
\hline Wadden [41] & $-6 \mathrm{~kg}$ & $-0.1 \mathrm{~kg}$ & $\leq 0.0001$ \\
\hline Blackman [44] & $-6.7 \mathrm{~kg}$ & $-1.9 \mathrm{~kg}$ & $\leq 0.0001$ \\
\hline Davies [42] & $-6 \mathrm{~kg}$ & $-2 \mathrm{~kg}$ & $\leq 0.001$ \\
\hline
\end{tabular}




\begin{tabular}{|c|c|c|c|}
\hline Pi-Sunyer [43] & $-8.4 \mathrm{~kg}$ & $-2.8 \mathrm{~kg}$ & $\leq 0.001$ \\
\hline Le Roux [46] & $-6.5 \mathrm{~kg}$ & $-2 \mathrm{~kg}$ & $\leq 0.0001$ \\
\hline Primary Author & Drug WC Difference & Control WC Difference & WC B/W Group P value \\
\hline Khoo [45] & $-5.4 \mathrm{~cm}$ & $-4.5 \mathrm{~cm}$ & NS \\
\hline Wadden [41] & $-4.7 \mathrm{~cm}$ & $-1.2 \mathrm{~cm}$ & $\leq 0.0001$ \\
\hline Blackman [44] & $-6.4 \mathrm{~cm}$ & $-3.1 \mathrm{~cm}$ & $\leq 0.0001$ \\
\hline Davies [42] & $-6.1 \mathrm{~cm}$ & $-2.7 \mathrm{~cm}$ & $\leq 0.001$ \\
\hline Pi-Sunyer [43] & $-8.2 \mathrm{~cm}$ & $-3.9 \mathrm{~cm}$ & $\leq 0.001$ \\
\hline Le Roux [46] & $-6.9 \mathrm{~cm}$ & $-3.4 \mathrm{~cm}$ & $\leq 0.0001$ \\
\hline Primary Author & Drug SBP Difference & Control SBP Difference & SBP B/W Group P value \\
\hline Wadden [41] & $0.2 \mathrm{mmHg}$ & $2.8 \mathrm{mmHg}$ & $\leq 0.01$ \\
\hline Blackman [44] & $-3.4 \mathrm{mmHg}$ & $0 \mathrm{mmHg}$ & $\leq 0.001$ \\
\hline Davies [42] & $-2.8 \mathrm{mmHg}$ & $-0.4 \mathrm{mmHg}$ & $\leq 0.01$ \\
\hline Pi-Sunyer [43] & $-4.2 \mathrm{mmHg}$ & $-1.5 \mathrm{mmHg}$ & $\leq 0.001$ \\
\hline Le Roux [46] & $-3.2 \mathrm{mmHg}$ & $-0.5 \mathrm{mmHg}$ & $\leq 0.0001$ \\
\hline Primary Author & Drug DBP Difference & Control DBP Difference & DBP B/W Group P value \\
\hline Wadden [41] & $1.4 \mathrm{mmHg}$ & $1.2 \mathrm{mmHg}$ & NS \\
\hline Blackman [44] & $-0.7 \mathrm{mmHg}$ & $-0.4 \mathrm{mmHg}$ & NS \\
\hline Davies [42] & $-0.9 \mathrm{mmHg}$ & $-0.5 \mathrm{mmHg}$ & NS \\
\hline Pi-Sunyer [43] & $-2.6 \mathrm{mmHg}$ & $-1.9 \mathrm{mmHg}$ & $\leq 0.001$ \\
\hline Le Roux [46] & $-2.3 \mathrm{mmHg}$ & $-1.9 \mathrm{mmHg}$ & NS \\
\hline Primary Author & D FBG Difference & C FBG Difference & FBG B/W Group P value \\
\hline Khoo [45] & $-12.6 \mathrm{mg} / \mathrm{dL}$ & $-10.8 \mathrm{mg} / \mathrm{dL}$ & NS \\
\hline Wadden [41] & $-9 \mathrm{mg} / \mathrm{dL}$ & $-3.6 \mathrm{mg} / \mathrm{dL}$ & $\leq 0.0001$ \\
\hline Blackman [44] & $-2.52 \mathrm{mg} / \mathrm{dL}$ & $-2.88 \mathrm{mg} / \mathrm{dL}$ & $\leq 0.0001$ \\
\hline Davies [42] & $-34.3 \mathrm{mg} / \mathrm{dL}$ & $-0.2 \mathrm{mg} / \mathrm{dL}$ & $\leq 0.001$ \\
\hline Pi-Sunyer [43] & $-7.1 \mathrm{mg} / \mathrm{dL}$ & $0.1 \mathrm{mg} / \mathrm{dL}$ & $\leq 0.001$ \\
\hline Le Roux [46] & $-6.66 \mathrm{mg} / \mathrm{dL}$ & $0.9 \mathrm{mg} / \mathrm{dL}$ & $\leq 0.0001$ \\
\hline Primary Author & D CRP (IQR) (mg/L) Difference & C CRP (IQR) (mg/L) Difference & $\begin{array}{c}\text { CRP (IQR) }(\mathrm{mg} / \mathrm{L}) \mathrm{B} / \mathrm{W} \text { Group P } \\
\text { value }\end{array}$ \\
\hline Khoo [45] & $-0.03 \mathrm{mg} / \mathrm{dL}$ & $-0.98 \mathrm{mg} / \mathrm{dL}$ & $\leq 0.01$ \\
\hline Wadden [41] & $-2.113 \mathrm{mg} / \mathrm{dL}$ & $0.1155 \mathrm{mg} / \mathrm{dL}$ & $\leq 0.01$ \\
\hline Blackman [44] & $-2.226 \mathrm{mg} / \mathrm{dL}$ & $-0.809 \mathrm{mg} / \mathrm{dL}$ & $\leq 0.05$ \\
\hline Davies [42] & $-1.13934 \mathrm{mg} / \mathrm{dL}$ & $-0.3762 \mathrm{mg} / \mathrm{dL}$ & $\leq 0.001$ \\
\hline Pi-Sunyer [43] & $-1.47 \mathrm{mg} / \mathrm{dL}$ & $-0.3838 \mathrm{mg} / \mathrm{dL}$ & $\leq 0.001$ \\
\hline Le Roux [46] & $-3.87 \mathrm{mg} / \mathrm{dL}$ & $-1.16 \mathrm{mg} / \mathrm{dL}$ & $\leq 0.0001$ \\
\hline Primary Author & Drug TC Difference & Control TC Difference & TC B/W Group P value \\
\hline Wadden [41] & $0.2 \mathrm{mg} / \mathrm{dL}$ & $0.3 \mathrm{mg} / \mathrm{dL}$ & NS \\
\hline Davies [42] & $-1.46 \mathrm{mg} / \mathrm{dL}$ & $3.8 \mathrm{mg} / \mathrm{dL}$ & $\leq 0.01$ \\
\hline Pi-Sunyer [43] & $-3.1 \mathrm{mg} / \mathrm{dL}$ & $-1 \mathrm{mg} / \mathrm{dL}$ & $\leq 0.001$ \\
\hline Primary Author & Drug LDL Difference & Control LDL Difference & LDL B/W Group P value \\
\hline Wadden [41] & $0.2 \mathrm{mg} / \mathrm{dL}$ & $0.3 \mathrm{mg} / \mathrm{dL}$ & NS \\
\hline Davies [42] & $0.58 \mathrm{mg} / \mathrm{dL}$ & $5.02 \mathrm{mg} / \mathrm{dL}$ & NS \\
\hline Pi-Sunyer [43] & $-3 \mathrm{mg} / \mathrm{dL}$ & $-1 \mathrm{mg} / \mathrm{dL}$ & $\leq 0.001$ \\
\hline
\end{tabular}




\begin{tabular}{|c|c|c|c|}
\hline Primary Author & Drug HDL Difference & Control HDL Difference & HDL B/W Group P value \\
\hline Wadden [41] & $0.2 \mathrm{mg} / \mathrm{dL}$ & $0.1 \mathrm{mg} / \mathrm{dL}$ & NS \\
\hline Davies [42] & $4.7 \mathrm{mg} / \mathrm{dL}$ & $1.93 \mathrm{mg} / \mathrm{dL}$ & $\leq 0.05$ \\
\hline Pi-Sunyer [43] & $2.3 \mathrm{mg} / \mathrm{dL}$ & $0.7 \mathrm{mg} / \mathrm{dL}$ & $\leq 0.001$ \\
\hline Primary Author & Drug TG Difference & Control TG Difference & TG B/W Group P value \\
\hline Wadden [41] & $0 \mathrm{mg} / \mathrm{dL}$ & $0.1 \mathrm{mg} / \mathrm{dL}$ & $\leq 0.05$ \\
\hline Davies [42] & $-14.68 \mathrm{mg} / \mathrm{dL}$ & $0.41 \mathrm{mg} / \mathrm{dL}$ & $\leq 0.001$ \\
\hline Pi-Sunyer [43] & $-13.3 \mathrm{mg} / \mathrm{dL}$ & $-5.5 \mathrm{mg} / \mathrm{dL}$ & $\leq 0.001$ \\
\hline
\end{tabular}

\section{Discussion}

Twenty-four studies of the five FDA-approved weight loss drugs demonstrated clinically significant effects on weight loss with differing effects on both cardiovascular and glycemic markers/risk factors. Saxenda ${ }^{\circledR}$, Belviq $^{\circledR}$, Contrave ${ }^{\circledR}$ and Qsymia ${ }^{\circledR}$ all positively affect fasting plasma glucose while Xenical ${ }^{\circledR}$ have no effect. Xenical ${ }^{\circledR}$, Qsymia $^{\circledR}$ and Contrave ${ }^{\circledR}$ demonstrated to have largely impacted cholesterol levels versus Belviq ${ }^{\circledR}$ and Saxenda ${ }^{\circledR}$ having minimal effects. Regarding systolic and diastolic blood pressure, Xenical ${ }^{\circledR}$, Qsymia $^{\circledR}$ and Saxenda ${ }^{\circledR}$ all showed positive effects compared to diet and exercise alone. According to Guideline for the Management of Overweight and Obesity in Adults, adjunctive pharmacotherapy should be considered for people who have either a BMI of 30 and over, or 27 and over with at least one comorbid conditions such as hypertension, dyslipidemia, type 2 diabetes or obstructive sleep apnea [14]. Even though it has been demonstrated that pharmacotherapy can enhance the likelihood of clinically meaningful weight loss and improve health, it is largely underutilized [17]. There is a potential role for pharmacist to be involved in primary care and serve as a liason between prescriber and weight management resources. Further research is needed to identify the cost effectiveness, most effective adjunctive behavioral treatments, the role of intermittent vs. continuous therapy as well as duration of treatment.

\section{Conclusion}

Underutilization of pharmacologic weight corrective therapies that have been statistically and clinically proven to be valuable tools in reducing obesity and its related risk factors [24]. Studies of the five FDA-approved drugs have demonstrated clinically significant effects on weight loss with differing effects on both cardiovascular and glycemic markers/risk factors.

\section{Author Contributions}

M.R. designed and directed the project; M.C. performed the experiments; M.H. wrote the manuscript with support from M.R. and M.C.

\section{References}

1. WHO Obesity and overweight fact sheet.

2. Wing RR, Bolin P, Brancati FL, Bray GA, Look AHEAD Research Group, et al. (2013) Cardiovascular effects of intensive lifestyle intervention in type 2 diabetes. N Engl J Med 369(2): 145-154.
3. Skolnik NS, Ryan DH (2014) Pathophysiology, epidemiology, and assessment of obesity in adults. J Fam Pract 63(7): S3-S10.

4. NHLBI/NIDDK (1998) Clinical guidelines on the identification, evaluation, and treatment of overweight and obesity in adults: The evidence report. National Institutes of Health: Bethesda, USA.

5. Thomas DM, Bouchard C, Church T, Kraus WE, Slentz C, et al. (2012) Why do individuals not lose more weight from an exercise intervention at a defined dose? An energy balance analysis. Obes Rev 13(10): 835-847.

6. Quetelet A (1835) On man and the development of his faculties, or essay in social physics. Paris.

7. Gordon T, Kannel WB (1976) Obesity and cardiovascular disease: The framingham study. Clin Endocrinol Metab 5(2): 367-375.

8. Rossner S (1991) Factors determining the long-term outcome of obesity treatment. In: Bjorntorp P, Brodoff BN (Eds.), Obesity. Philadelphia: J B Lippincott Co, USA. pp. 712-719.

9. Goldstein DJ (1992) Beneficial health effects of modest weight loss. Int J Obes 16(6): 397-415.

10. Blackburn G (1995) Effect of degree of weight loss on health benefits. Obes Res 3(2): s211-s216.

11. Knowler WC, Connor EB, Fowler SE, Hamman RF, Diabetes Prevention Program Research Group (2002) Reduction in the incidence of type 2 diabetes with lifestyle intervention or metformin. New Engl J Med 346: 393-403.

12. Douketis JD, Macie C, Thabane L, Williamson DF (2005) Systematic review of long-term weight loss studies in obese adults: Clinical significance and applicability to clinical practice. Int J Obes 29(10): 1153-1167.

13. Hamman RF, Wing RR, Edelstein SL, Lachin JM, Bray GA, et al. (2006) Effect of weight loss with lifestyle intervention on risk of diabetes. Diabetes Prevention Program Research Group. Diabetes Care 29(9): 2102-2107.

14. Jensen MD, Ryan DH, Donato KA (2014) Guidelines (2013) for managing overweight and obesity in adults. Obesity 22: S1-S410.

15. Wing RR, Lang W, Wadden TA, Safford M, Look AHEAD Research Group, et al. (2011) Benefits of modest weight loss in improving cardiovascular risk factors in overweight and obese individuals with type 2 diabetes. Diabetes Care 34(7): 1481-1486.

16. Semla TP, Ruser C, Good CB, Yanovski SZ, Ames D, et al. (2017) Pharmacotherapy for weight management in the VHA. J Gen Intern Med 32(Suppl 1): 70-73.

17. Hampp C, Kang EM, Borders Hemphill V (2013) Use of prescription antiobesity drugs in the United States. Pharmacotherapy 33(12): 12991307.

18. (2018) Xenical (orlistat). Montgomery, AL: H2-Pharma LLC., USA.

19. (2018) Qsymia (phentermine and topiramate). Mountain View, CA: VIVUS Inc., USA. 
20. (2018) Belviq (lorcaserin). Woodcliff Lake, NJ: Eisai Inc., Japan.

21. (2018) Contrave (naltrexone and bupropion). La Jolla, CA: Orexigen Therapeutics Inc., USA.

22. (2018) Saxenda (liraglutide). Plainsboro, NJ: Novo Nordisk, Denmark.

23. Davidson MH, Hauptman J, Digirolamo M, Foreyt JP, Halsted CH, et al. (1999) Effects of a weight-reduction program with orlistat on serum leptin levels in obese women: A 12-week, randomized, placebocontrolled study. JAMA 281(3): 235-242.

24. Finer N, James WPT, Kopelman PG, Lean ME, Williams G (2000) One-year treatment of obesity: A randomized, double-blind, placebo-controlled, multicenter study of orlistat, a gastrointestinal lipase inhibitor. International Journal of Obesity 24(3): 306-313.

25. Muls E, Kolanowski J, Scheen A, Van Gaal L, ObelHyx Study Group (2001) The effects of orlistat on weight and on serum lipids in obese patients with hypercholesterolemia: A randomized, double-blind, placebocontrolled, multicentre study. International Journal of Obesity 25(11): 1713-1721.

26. Ozcelik O, Dogan H, Kelestimur H (2004) Effects of a weight-reduction program with orlistat on serum leptin levels in obese women: A 12week, randomized, placebo-controlled study. Current Therapeutic Research 65(2): 127-137.

27. Torgerson JS, Hauptan J, Boldrin MN, Sjöström L (2004) XENical in the prevention of diabetes in obese subjects (XENDOS) study: A randomized study of orlistat as an adjunct to lifestyle changes for the prevention of type 2 diabetes in obese patients. Diab Care 27(1): 155-161.

28. Kujawska Luczak M, Musialik K, Szulinska M, Cwynar ES, Kargulewicz A et al. (2017) The effect of orlistat versus metformin on body composition and insulin resistance in obese premenopausal women: 3-month randomized prospective open-label study. Arch Med Sci 13(4): 725-731.

29. Allison DB, Gadde KM, Garvey WT, Peterson CA, Schwiers ML, et al. (2011) Controlled-release phentermine/topiramate in severely obese adults: A randomized controlled trial (EQUIP). Obesity 20(2): 330-342.

30. Gadde KM, Allison DB, Ryan DH, Peterson CA, Troupin B, et al. (2011) Effects of low-dose, controlled-release, phentermine plus topiramate combination on weight and associated comorbidities in overweight and obese adults (CONQUER): A randomized, placebo-controlled, phase 3 trial. Lancet 377(9774): 1341-1352.

31. Garvey WT, Ryan DH, Look M, Gadde KM, Allison DB, et al. (2012) Twoyear sustained weight loss and metabolic benefits with controlledrelease phentermine/topiramate in obese and overweight adults (SEQUEL): A randomized, placebo-controlled, phase 3 extension study. Am J Clin Nutr 95(2): 297-308.

32. Winslow DH, Bowden CH, Didonato KP, McCullough PA (2012) A randomized, double-blind, placebo-controlled study of an oral, extended release formulation of phentermine/topiramate for the treatment of obstructive sleep apnea in obese adults. Sleep 35(11): 1529-1539.

33. Fidler MC, Sanchez M, Raether B, Weissman NJ, Smith SR, et al. (2011) A one-year randomized trial of lorcaserin for weight loss in obese and overweight adults: The BLOSSOM Trial. J Clin Endocrinol Metab 96(10): 3067-3077.
34. O Neil PM, Smith SR, Weissman NJ, Fidler MC, Sanchez M, et al. (2012) Randomized placebo-controlled clinical trial of lorcaserin for weight loss in type 2 diabetes mellitus: The BLOOM-DM Study. Obesity 20(7): 1426-1436.

35. Smith SR, Garvey WT, Greenway FL, Zhou S, Fain R, et al. (2017) Coadministration of lorcaserin and phentermine for weight management: A 12-week, randomized, pilot safety study. Obesity 25(5): 857-865.

36. Tronieri JS, Wadden TA, Berkowitz RI, Chao AM, Pearl RL, et al. (2018) A randomized trial of lorcaserin and lifestyle counseling for maintaining weight loss achieved with a low-calorie diet. Obesity 26(2): 299-309.

37. Greenway FL, Dunayevich E, Tollefson G, Erickson J, Guttadauria M, et al. (2009) Comparison of combined bupropion and naltrexone therapy for obesity with monotherapy and placebo. J Clin Endocrinol Metab 94(12): 4898-4906.

38. Greenway FL, Fujioka K, Plodkowski RA, Mudaliar S, Guttadauria M, et al. (2010) Effect of naltrexone plus bupropion on weight loss in overweight and obese adults (COR-I): A multicenter, randomized, double-blind, placebo-controlled, phase 3 trial. Lancet 376(9741): 595-605.

39. Apovian CM, Aronne L, Rubino D, Still C, Wyatt H, et al. (2013) A randomized, phase 3 trial of naltrexone SR/bupropion SR on weight and obesity-related risk factors (COR-II). Obesity 21(5): 935-943.

40. Nissen SE, Wolski KE, Prcela L, Wadden T, Buse JB, et al. (2016) Effect of naltrexone-bupropion on major adverse cardiovascular events in overweight and obese patients with cardiovascular risk factors: A randomized clinical trial. JAMA 315(10): 990-1004.

41. Wadden TA, Hollander P, Klein S, Niswender K, Woo V, et al. (2013) Weight maintenance and additional weight loss with liraglutide after low-calorie-diet-induced weight loss: The SCALE Maintenance randomized study. Intern J Obesity 37(11): 1443-1451.

42. Davies MJ, Bergenstal R, Bode B, Kushner RF, Lewin A, et al. (2015) Efficacy of liraglutide for weight loss among patients with type 2 diabetes: The SCALE diabetes randomized clinical trial. JAMA 314(7): 687-699.

43. Pi Sunyer X, Astrup A, Fujioka K, Halpern A, Krempf M, et al. (2015) A randomized, controlled trial of $3.0 \mathrm{mg}$ of liraglutide in weight management. N Engl J Med 373(1): 11-22.

44. Blackman A, Foster GD, Zammit G, Rosenberg R, Aronne L, et al. (2016) Effect of liraglutide $3.0 \mathrm{mg}$ in individuals with obesity and moderate or severe obstructive sleep apnea: The SCALE sleep apnea randomized clinical trial. Int J Obes 40(8): 1310-1319.

45. Khoo J, Hsiang J, Taneja R, Law NM, Ang TL, et al. (2017) Comparative effects of liraglutide $3 \mathrm{mg}$ vs structured lifestyle modification on body weight, liver fat and liver function in obese patients with non-alcoholic fatty liver disease: A pilot randomized trial. Diabetes Obes Metab 19(12): 1814-1817.

46. Le Roux CW, Astrup A, Fukioka K, Greenway F, Lau DCW, et al. (2017) 3 years of liraglutide versus placebo for type 2 diabetes risk reduction and weight management in individuals with prediabetes: A randomised, double-blind trial. Lancet 389(10077): 1399-1409. 